\title{
Cohomology and duality for coalgebras over a quadratic operad
}

\author{
Anita MAJUMDAR ${ }^{a}$ and Donald $Y A U^{b}$ \\ a Department of Mathematics, Indian Institute of Science, Bangalore 560012, India \\ E-mail: anita@math.iisc.ernet.in \\ ${ }^{b}$ Department of Mathematics, The Ohio State University at Newark, \\ 1179 University Drive, Newark, OH 43055, USA \\ E-mail:dyau@math.ohio-state.edu
}

\begin{abstract}
The cohomology of a finite-dimensional coalgebra over a finitely generated quadratic operad, with coefficients in itself, is defined and is shown to have the structure of a graded Lie algebra. The cohomology of such a coalgebra is isomorphic to the cohomology of its linear dual as graded Lie algebras.
\end{abstract}

2000 MSC: 18D50, 20G10, 16W30

\section{Introduction}

Operads are mathematical devices that encode algebraic structures. They were originally introduced by May [24] to study iterated loop spaces in algebraic topology. Operads have since been used with great success in many other fields, including algebra, mathematical physics, and computer science. See [23] for a general survey of generalities and applications of operads. In particular, familiar structures such as associative algebras, Lie algebras, commutative algebras, and Gerstenhaber algebras are all algebras over some corresponding operads.

Let $A$ be an associative algebra, and let $C$ be a coassociative coalgebra. The structure of the Hochschild cohomology [11] $H_{h}^{*}(A, A)$ of $A$ with self-coefficients is well known; it is a Gerstenhaber algebra [6]. The same is true for the Hochschild coalgebra cohomology $H_{c}^{*}(C, C)$ [12], which can be proved by basically the same arguments as in [6]. If, in addition, $A$ is finitedimensional, then $H_{h}^{*}(A, A)$ and $H_{c}^{*}\left(A^{\#}, A^{\#}\right)$ are isomorphic as graded vector spaces [26], where $A^{\#}$ is the dual coalgebra of $A$.

In this paper, we generalize this duality isomorphism to finite-dimensional (co)algebras over any finitely generated quadratic operad $\mathcal{P}$. In particular, we will do the following:

(1) Define cohomology $\bar{H}_{\mathcal{P}}^{*}(V)$ with self-coefficients for finite-dimensional $\mathcal{P}$-coalgebras $V$.

(2) Observe that $\bar{H}_{\mathcal{P}}^{*}(V)$ is a graded Lie algebra, whose graded Lie bracket is induced by one on the defining cochain complex $\bar{C}_{\mathcal{P}}^{*}(V)$.

(3) Show that $\bar{H}_{\mathcal{P}}^{*}(V)$ and $H_{\mathcal{P}}^{*}\left(V^{\#}\right)$ are isomorphic as graded Lie algebras, and vice versa, where $H_{\mathcal{P}}^{*}\left(V^{\#}\right)$ is the cohomology (as defined in [2]) of the dual $\mathcal{P}$-algebra $V^{\#}$ of $V$.

We note that the differential graded Lie algebra $\bar{C}_{\mathcal{P}}^{*}(V)$ controls the deformations of $V$ as a $\mathcal{P}$ coalgebra in the sense of Gerstenhaber [7].

When $\mathcal{P}$ is the associative algebra operad As, the cohomology $\bar{H}_{\mathbf{A s}}^{*}(V)$ (resp., $H_{\mathbf{A} \mathbf{s}}^{*}(V)$ ) coincides with $H_{c}^{*}(V, V)$ (resp., $H_{h}^{*}(V, V)$ ). In this case, we recover the duality isomorphism 
in the Hochschild case discussed above, strengthened with the graded Lie brackets. Taking other finitely generated quadratic operads $\mathcal{P}$, the above statements apply to other classical (co)algebras, including commutative [10], Lie [4], Poisson, and Gerstenhaber (co)algebras. They also cover, for example, the cases of Leibniz (co)algebras [14, 15, 16, 18], Loday-type (co)algebras $[1,5,17,19,20,21,27,28]$, and ennea-(co)algebras [13].

\subsection{Organization}

In the next section, we recall from $[2,3]$ the constructions and some properties of the cochain complex $C_{\mathcal{P}}^{*}(A)$ that defines the cohomology $H_{\mathcal{P}}^{*}(A)$ with self-coefficients of a $\mathcal{P}$-algebra $A$.

In Section 3, we study properties of $\mathcal{P}$-coalgebras. In particular, it is observed (Theorem 3.3) that a $\mathcal{P}$-coalgebra structure is equivalent to a degree 1 differential derivation on the free graded $\mathcal{P}^{!}$-algebra generated by $V$, where $\mathcal{P}^{!}$denotes the Koszul dual operad of $\mathcal{P}$.

In Section 4 , the cochain complex $\bar{C}_{\mathcal{P}}^{*}(V)$ that defines the cohomology $\bar{H}_{\mathcal{P}}^{*}(V)$ of a finitedimensional $\mathcal{P}$-coalgebra $V$ is defined. It is first shown to be a graded Lie algebra (Corollary 4.3). A $\mathcal{P}$-coalgebra structure can be characterized as a square-zero element in this graded Lie algebra (Corollary 4.5). The differential $\bar{\delta}_{\pi}$ on $\bar{C}_{\mathcal{P}}^{*}(V)$ is then defined as, up to a nonzero scalar multiple, the inner derivation $[-, \pi]$ with respect to the square-zero element $\pi$ that defines the $\mathcal{P}$-coalgebra structure on $V(4.3)$. This makes $\bar{C}_{\mathcal{P}}^{*}(V)$ into a differential graded Lie algebra (Corollary 4.6), which implies that $\bar{H}_{\mathcal{P}}^{*}(V)$ is a graded Lie algebra (Corollary 4.7). Example 4.8 makes this explicit in the case of the Hochschild coalgebra cochain complex $\bar{C}_{\mathbf{A s}}^{*}(V)=\operatorname{Hom}\left(V, V^{\otimes *}\right)$.

In Section 5, it is shown that, for a finite-dimensional $\mathcal{P}$-coalgebra $V$, the differential graded Lie algebras $\bar{C}_{\mathcal{P}}^{*}(V)$ and $C_{\mathcal{P}}^{*}\left(V^{\#}\right)$ are isomorphic (Corollary 5.3). The duality isomorphism between $\bar{H}_{\mathcal{P}}^{*}(V)$ and $H_{\mathcal{P}}^{*}\left(V^{\#}\right)$ is then an immediate consequence after passing to cohomology (Corollary 5.4). The special case involving Hochschild (coalgebra) cohomology is explained in Example 5.5.

In Section 6, an explicit description of the differential $\bar{\delta}_{\pi}$ in $\bar{C}_{\mathcal{P}}^{*}(V)$ is given in terms of the

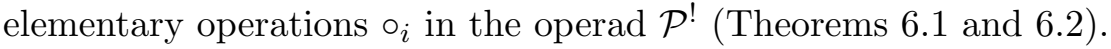

\section{Algebras over an operad}

In this section, we recall some basic definitions and results about operads and the cohomology of an algebra over a quadratic operad with coefficients in itself.

\subsection{Conventions}

The symbol $\mathbb{N}^{*}$ denotes the set of positive integers. Throughout this paper, we work over a fixed field $\mathbf{k}$ of characteristic zero. Vector spaces, $\otimes$, Hom, and End (endomorphisms) are all meant over k. For any positive integer $n, \Sigma_{n}$ will denote the group of permutations on $n$ elements. For $\sigma \in \Sigma_{n}, \epsilon(\sigma) \in\{-1,1\}$ will stand for the sign of $\sigma$, and $\mathbf{s g n}_{n}$ will denote the sign representation of $\Sigma_{n}$.

\subsection{Operads}

An operad $[22,23,24,25] \mathcal{P}$ consists of a right $\mathbf{k}\left[\Sigma_{n}\right]$-module $\mathcal{P}(n)$, one for each $n \in \mathbb{N}^{*}$. For positive integers $n, j_{1}, \ldots, j_{n}$, there is a structure map

$$
\gamma: \mathcal{P}(n) \otimes \mathcal{P}\left(j_{1}\right) \otimes \cdots \otimes \mathcal{P}\left(j_{n}\right) \rightarrow \mathcal{P}\left(j_{1}+\cdots+j_{n}\right)
$$


These structure maps satisfy some associativity, equivariance, and unity conditions, which can be found in [24]. Using the operad structure maps, one defines the $\circ_{i}$ operations as

$$
f \circ_{i} g=\gamma(f ; 1, \ldots, 1, g, 1, \ldots, 1) \in \mathcal{P}(n+m-1)
$$

for $f \in \mathcal{P}(n)$ and $g \in \mathcal{P}(m)$, where there are $(i-1)$ copies of 1's in front of $g$. Conversely, the structure maps $\gamma$ can be recovered from the $\circ_{i}$ operations as

$$
\gamma\left(f ; g_{1}, \ldots, g_{n}\right)=\left(\cdots\left(\left(\left(f \circ_{1} g_{1}\right) \circ_{j_{1}+1} g_{2}\right) \circ_{j_{1}+j_{2}+1} g_{3}\right) \cdots\right)
$$

for $f \in \mathcal{P}(n)$ and $g_{i} \in \mathcal{P}\left(j_{i}\right)(1 \leq i \leq n)$. In the presence of the unit $1 \in \mathcal{P}(1)$, the operad structure maps $\gamma$ are completely determined by the $\circ_{i}$ operations (see [22] or [23, Section 1.7.1, p. 66]). In what follows, by using (2.1) and (2.2), we will use these two equivalent definitions of an operad interchangeably.

\subsection{Non- $\Sigma$ operads}

From the definition of an operad, if we omit the parts concerning the symmetric groups $\Sigma_{n}$ $(n \geq 1)$, then we obtain the definition of a non- $\Sigma$ operad.

\subsection{Operad morphisms}

Let $\mathcal{P}$ and $\mathcal{Q}$ be two operads. A morphism of operads from $\mathcal{P}$ to $\mathcal{Q}$ is a sequence $a=\{a(n), n \in$ $\left.\mathbb{N}^{*}\right\}$ of $\mathbf{k}\left[\Sigma_{n}\right]$-linear maps $a(n): \mathcal{P}(n) \rightarrow \mathcal{Q}(n)$ satisfying the conditions $a(1)(1)=1$ and $a(n+$ $m-1)\left(\mu \circ_{i} \nu\right)=a(n)(\mu) \circ_{i} a(m)(\nu)$ for $n, m \in \mathbb{N}^{*}, 1 \leq i \leq n, \mu \in \mathcal{P}(n)$, and $\nu \in \mathcal{P}(m)$.

\subsection{Endomorphism operad}

Let $V$ be a vector space over $\mathbf{k}$. For $n \in \mathbb{N}^{*}$, let $\operatorname{End}(V)(n)=\operatorname{Hom}\left(V^{\otimes n}, V\right)$. Then $\operatorname{End}(V)=$ $\left\{\operatorname{End}(V)(n), n \in \mathbb{N}^{*}\right\}$ is naturally an operad under composition, called the endomorphism operad of $V$. Indeed, $f \circ \circ_{i} g=f(1, \ldots, g, \ldots, 1)$, where $g$ is in the $i$ th place, and 1 is the identity map on $V$. The $\Sigma_{n}$-action on $\operatorname{End}(V)(n)$ comes from composition with the left $\Sigma_{n}$-action on $V^{\otimes n}$.

\subsection{Algebras over an operad}

Let $\mathcal{P}$ be an operad. A $\mathcal{P}$-algebra or an algebra over $\mathcal{P}$ is a vector space $V$ over $\mathbf{k}$ along with a morphism of operads $\pi: \mathcal{P} \rightarrow \operatorname{End}(V)$. Using adjunctions, a $\mathcal{P}$-algebra structure on $V$ can be expressed in terms of maps $\pi_{n}: \mathcal{P}(n) \otimes_{\Sigma_{n}} V^{\otimes n} \rightarrow V$ that satisfy the obvious associativity, equivariant, and unity conditions.

Proposition 2.1 (see [9]). Let $E$ be a right $\mathbf{k}\left[\Sigma_{2}\right]$-module. Then there exists an operad $\mathcal{F}(E)$ with $\mathcal{F}(E)(1)=\mathbf{k}$ and $\mathcal{F}(E)(2)=E$ such that the following property holds: for any operad $\mathcal{Q}$ and for any morphism of right $\mathbf{k}\left[\Sigma_{2}\right]$-modules $a: E \rightarrow \mathcal{Q}(2)$, there exists a unique morphism of operads, $\hat{a}: \mathcal{F}(E) \rightarrow \mathcal{Q}$, such that $\hat{a}(2)=a$.

\section{$2.7 \quad$ Free operad}

The operad $\mathcal{F}(E)$ is called the free operad generated by $E$. By the usual arguments, the free operad $\mathcal{F}(E)$ is unique up to operad isomorphisms. 


\subsection{Free graded $\mathcal{P}$-algebra}

For an operad $\mathcal{P}$ and a vector space $V$, define the free graded $\mathcal{P}$-algebra generated by $V$ as

$$
\mathcal{F}_{\mathcal{P}}^{g r}(V)=\oplus_{n \geq 1} \mathcal{P}(n) \otimes_{\Sigma_{n}}\left(V^{\otimes n} \otimes \mathbf{s g n}_{n}\right)
$$

where $\sigma\left(v_{1} \otimes \cdots \otimes v_{n}\right)=\epsilon(\sigma) v_{\sigma^{-1}(1)} \otimes \cdots \otimes v_{\sigma^{-1}(n)}$ for $\sigma \in \Sigma_{n}$ and $v_{i} \in V$. The homogeneous degree $n$ component of $\mathcal{F}_{\mathcal{P}}^{g r}(V)$ is denoted by $\mathcal{F}_{\mathcal{P}}^{g r_{n}}(V)$. The $\mathcal{P}$-algebra structure on $\mathcal{F}_{\mathcal{P}}^{g r}(V)$ is the natural one defined by the operad structure on $\mathcal{P}$ and concatenation on $V^{\otimes *}[3,1.6 .2]$.

\subsection{Graded derivations}

Let $A=\oplus_{j \geq 0} A^{j}$ be a graded algebra over an operad $\mathcal{P}$ with structure maps $\pi_{m}: \mathcal{P}(m) \otimes \Sigma_{m}$ $A^{\otimes m} \rightarrow A$. A degree $n$ derivation of $A$ is a homogeneous degree $n$ linear map $d: A \rightarrow A$ such that

$$
d\left(\pi_{m}\left(\mu ; a_{1}, \ldots, a_{m}\right)\right)=\sum_{i=1}^{m}(-1)^{n s_{i}} \pi_{m}\left(\mu ; a_{1}, \ldots, a_{i-1}, d\left(a_{i}\right), a_{i+1}, \ldots, a_{m}\right)
$$

for $m \in \mathbb{N}^{*}, \mu \in \mathcal{P}(m)$, and $a_{i} \in A$. Here $s_{i}=\left|a_{1}\right|+\cdots+\left|a_{i-1}\right|$ with $s_{1}=0$, and $|a|=j$ if $a \in A^{j}\left[3\right.$, Definition 2.3.1]. The set of degree $n$ derivations of $A$ is denoted by $\operatorname{Der}^{n}(A)$. Denote by $\operatorname{Der}(A)$ the graded vector space $\oplus_{n \geq 0} \operatorname{Der}^{n}(A)$ of all derivations of $A$.

\subsection{Operadic ideals}

Let $\mathcal{P}$ be an operad. An ideal of $\mathcal{P}$ is a sequence $I=\left\{I(n), n \in \mathbb{N}^{*}\right\}$, in which $I(n)$ is a $\mathbf{k}\left[\Sigma_{n}\right]$ submodule of $\mathcal{P}(n)$, such that for $\mu \in \mathcal{P}(n), \nu \in \mathcal{P}(m), x \in I(m), y \in I(n)$, and $1 \leq i, j \leq n$, one has that $\mu \circ_{i} x \in I(n+m-1)$ and $y \circ_{j} \nu \in I(n+m-1)$.

When $I$ is an ideal of $\mathcal{P}$, the quotient $\mathcal{P} / I=\{(\mathcal{P} / I)(n)=\mathcal{P}(n) / I(n)\}$ inherits an operad structure from $\mathcal{P}$.

\subsection{Quadratic operads}

Let $E$ be a right $\mathbf{k}\left[\Sigma_{2}\right]$-module and let $R$ be a right $\mathbf{k}\left[\Sigma_{3}\right]$-submodule of $\mathcal{F}(E)(3)$. Let $(R)$ be the ideal generated by $R$. Then the quotient operad $\mathcal{F}(E) /(R)$ is called the quadratic operad generated by $E$ with relations $R$, denoted by $\mathcal{P}(\mathbf{k}, E, R)$ [9]. A quadratic operad $\mathcal{P}(\mathbf{k}, E, R)$ is said to be finitely generated if $E$ is a finite-dimensional vector space.

Proposition 2.2 (see [3, Proposition 1.5.5]). Let $\mathcal{P}=\mathcal{P}(\mathbf{k}, E, R)$ be a quadratic operad. Then a $\mathcal{P}$-algebra structure on a vector space $V$ is determined by a morphism of right $\mathbf{k}\left[\Sigma_{2}\right]$-modules $\pi: \mathcal{P}(2)=E \rightarrow \operatorname{End}(V)(2)$ such that $\hat{\pi}(3)(R)=0$.

In this case, the morphism $\pi: \mathcal{P}(2) \rightarrow \operatorname{End}(V)(2)$, or equivalently its adjoint $\pi: \mathcal{P}(2) \otimes_{\Sigma_{2}}$ $V^{\otimes 2} \rightarrow V$, is called the structural morphism of the $\mathcal{P}$-algebra $V$.

\subsection{Quadratic duality}

Let $F$ be a right $\mathbf{k}\left[\Sigma_{n}\right]$-module. By $F^{\#}$ we mean the right $\mathbf{k}\left[\Sigma_{n}\right]$-module $F^{\#}=\operatorname{Hom}(F, \mathbf{k}) \otimes \mathbf{s g n}_{n}$, where the right $\Sigma_{n}$-action is given by $(\phi \cdot \sigma)(x)=\epsilon(\sigma) \phi\left(x \cdot \sigma^{-1}\right)$ for $\phi \in \operatorname{Hom}(F, \mathbf{k})$ and $x \in F$. 
Let $E$ be a right $\mathbf{k}\left[\Sigma_{2}\right]$-module. Then as right $\mathbf{k}\left[\Sigma_{3}\right]$-modules, one has that $[9] \mathcal{F}\left(E^{\#}\right)(3) \cong$ $(\mathcal{F}(E)(3))^{\#}$. Let $R \subset \mathcal{F}(E)(3)$ be a right $\mathbf{k}\left[\Sigma_{3}\right]$-submodule, and let $R^{\perp} \subset \mathcal{F}\left(E^{\#}\right)(3)$ be the annihilator of $R$ in $(\mathcal{F}(E)(3))^{\#} \cong \mathcal{F}\left(E^{\#}\right)(3)$. The Koszul dual of the quadratic operad $\mathcal{P}=$ $\mathcal{P}(\mathbf{k}, E, R)$ is defined as the quadratic operad $\mathcal{P}^{!}=\mathcal{P}\left(\mathbf{k}, E^{\#}, R^{\perp}\right)$.

\subsection{The graded Lie algebra $L_{\mathcal{P}}(V)$}

We briefly recall the cohomology of an algebra over a finitely generated quadratic operad, due to Balavoine $[2,3]$.

For the rest of this section, let $\mathcal{P}=\mathcal{P}(\mathbf{k}, E, R)$ be a finitely generated quadratic operad, and let $V$ be a finite-dimensional vector space. To simplify notations, let $\left(\mathcal{P}^{!}\right)^{\#}(n)$ stand for $\left(\mathcal{P}^{!}(n)\right)^{\#}$. Define the vector spaces

$$
\begin{aligned}
& L_{\mathcal{P}}^{n}(V)=\mathcal{P}^{!}(n+1) \otimes_{\Sigma_{n+1}} \overline{\operatorname{End}}(V)(n+1) \quad(n \geq 0) \\
& C_{\mathcal{P}}^{n}(V)=\operatorname{Hom}\left(\left(\mathcal{P}^{!}\right)^{\#}(n) \otimes_{\Sigma_{n}} V^{\otimes n}, V\right) \quad(n \geq 1)
\end{aligned}
$$

where $\overline{\operatorname{End}}(V)(n)=\operatorname{End}(V)(n) \otimes \mathbf{s g n}_{n}$, with the natural structure of a left $\mathbf{k}\left[\Sigma_{n}\right]$-module. Let $L_{\mathcal{P}}(V)$ denote the graded vector space $L_{\mathcal{P}}(V)=\bigoplus_{n \geq 0} L_{\mathcal{P}}^{n}(V)$. Using the elementary operations ${ }_{i}$ in the operad $\mathcal{P}^{!}$and the non- $\Sigma$ operad $\overline{\operatorname{End}}(V)=\oplus_{n \geq 1} \overline{\operatorname{End}}(V)(n)$, one defines the operations $[2,3]$

$$
\begin{aligned}
\left(\mu^{*} \otimes f\right) \circ\left(\nu^{*} \otimes g\right) & =\sum_{i=1}^{n+1}(-1)^{m(i-1)}\left(\mu^{*} \circ_{i} \nu^{*}\right) \otimes\left(f \circ_{i} g\right) \\
{\left[\mu^{*} \otimes f, \nu^{*} \otimes g\right] } & =\left(\mu^{*} \otimes f\right) \circ\left(\nu^{*} \otimes g\right)+(-1)^{n m+1}\left(\nu^{*} \otimes g\right) \circ\left(\mu^{*} \otimes f\right)
\end{aligned}
$$

for $\mu^{*} \otimes f \in L_{\mathcal{P}}^{n}(V)$ and $\nu^{*} \otimes g \in L_{\mathcal{P}}^{m}(V)$. These two operations are indeed well defined, i.e., independent of the choice of representing elements $\mu^{*} \otimes f$ and $\nu^{*} \otimes g$. However, the individual ${ }^{\circ}{ }_{i}$ operations are not well defined on $L_{\mathcal{P}}(V)$ [3, Remark 2.4.4].

Proposition 2.3 (see [3, Proposition 2.4.4]). The bracket $[-,-]$ defined above makes $L_{\mathcal{P}}(V)$ into a graded Lie algebra.

Indeed, once one establishes that $\circ$ and $[-,-]$ are well defined, this result follows from $[8,(3)]$, since $\mathcal{P}^{!} \otimes \overline{\operatorname{End}}(V)$ is a non- $\Sigma$ operad.

Proposition 2.4 (see [2, Proposition 3.1.4]). There is an isomorphism of vector spaces

$$
\Gamma: L_{\mathcal{P}}^{n}(V) \stackrel{\cong}{\longrightarrow} C_{\mathcal{P}}^{n+1}(V)
$$

With the obvious notations, the isomorphism $\Gamma$ is given by

$$
\Gamma\left(\mu^{*} \otimes f\right)(\mu \otimes x)=\frac{1}{n !} \sum_{\sigma \in \Sigma_{n}} \epsilon(\sigma)\left\langle\mu, \mu^{*} \sigma\right\rangle f(\sigma x)
$$




\subsection{The graded Lie algebra $C_{\mathcal{P}}^{*}(V)$}

Using the isomorphism $\Gamma$, the graded Lie bracket on $L_{\mathcal{P}}(V)$ can be transported to $C_{\mathcal{P}}^{*}(V)$, which makes $C_{\mathcal{P}}^{*}(V)$ into a graded Lie algebra of degree -1 . More precisely, the graded Lie bracket on $C_{\mathcal{P}}^{*}(V)$ is defined as

$$
[f, g]=\Gamma\left(\left[\Gamma^{-1}(f), \Gamma^{-1}(g)\right]\right)
$$

The same can be said of the operation $\circ$, from which the graded Lie bracket is defined.

Proposition 2.5 (see [3, Proposition 2.3.3]). There is an isomorphism of vector spaces

$$
\omega: \operatorname{Der}^{n}\left(\mathcal{F}_{\mathcal{P}^{!}}^{g r}\left(V^{\#}\right)\right) \stackrel{\cong}{\longrightarrow} C_{\mathcal{P}}^{n+1}(V)
$$

The space $\operatorname{Der}\left(\mathcal{F}_{\mathcal{P} !}^{g r}\left(V^{\#}\right)\right)$ of derivations has a natural graded Lie bracket, namely, the commutator bracket. Using the isomorphism $\omega$, this gives rise to another graded Lie bracket of degree -1 on $C_{\mathcal{P}}^{*}(V)$. These two graded Lie brackets on $C_{\mathcal{P}}^{*}(V)$ are equal $[3,2.4 .4]$.

Theorem 2.6 (see [3, Theorem 2.4.1 and Corollary 2.4.2]). The following three sets are in bijection with each other:

(1) The set of $\mathcal{P}$-algebra structures on $V$.

(2) The set of degree 1 derivations $d \in \operatorname{Der}^{1}\left(\mathcal{F}_{\mathcal{P} !}^{g r}\left(V^{\#}\right)\right)$ that satisfy $d^{2}=0$.

(3) The set of elements $\pi \in C_{\mathcal{P}}^{2}(V)$ that satisfy $[\pi, \pi]=0$.

When one of these equivalent conditions is satisfied, we say that $(V, \pi)$ is a $\mathcal{P}$-algebra.

Note that $[\pi, \pi]=0$ is equivalent to the condition $\hat{\pi}(3)(R)=0$ in Proposition 2.2.

\subsection{Cohomology of a $\mathcal{P}$-algebra}

Let $(A, \pi)$ be a $\mathcal{P}$-algebra. Define the map $\delta_{\pi}^{n}: C_{\mathcal{P}}^{n}(A) \rightarrow C_{\mathcal{P}}^{n+1}(A)$ by setting

$$
\delta_{\pi}^{n}(f)=-\frac{n+1}{2}[f, \pi]
$$

By [2, Proposition 3.1.7], the map $\delta_{\pi}^{*}$ is a differential. The homology of the cochain complex $\left(C_{\mathcal{P}}^{*}(A), \delta_{\pi}^{*}\right)$ is denoted by $H_{\mathcal{P}}^{*}(A)$ or $H_{\mathcal{P}}^{*}(A, \pi)$, and it is called the cohomology of the $\mathcal{P}$-algebra $A$ with coefficients in itself or simply the operadic cohomology of $A$. With the induced Lie bracket, $H_{\mathcal{P}}^{*}(A)$ becomes a graded Lie algebra. Also, note that $\left(C_{\mathcal{P}}^{*}(V), \delta_{\pi},[-,-]\right)$ is a differential graded

Lie algebra, which controls the deformations of $V$ as a $\mathcal{P}$-algebra [3, Section 4$]$ in the sense of Gerstenhaber [7].

\section{Coalgebras over an operad}

The purpose of this section is to give alternative characterizations of $\mathcal{P}$-coalgebra structures on a finite-dimensional vector space in terms of differential derivations when $\mathcal{P}$ is a finitely generated quadratic operad. 


\subsection{Coendomorphism operad}

Let $V$ be a vector space. Let $\operatorname{Coend}(V)=\left\{\operatorname{Hom}\left(V, V^{\otimes n}\right)\right\}$ be the coendomorphism operad of $V$ with the obvious structure maps, dual to those in $\operatorname{End}(V)$. For an operad $\mathcal{P}$, a $\mathcal{P}$-coalgebra structure on $V$ is a morphism $\mathcal{P} \rightarrow \operatorname{Coend}(V)$ of operads.

For example, a coassociative coalgebra structure is equivalent to an As-coalgebra structure, where $\mathbf{A s}$ is the associative algebra operad.

Proposition 3.1. Let $\mathcal{P}=\mathcal{P}(\mathbf{k}, E, R)$ be a finitely generated quadratic operad, and let $V$ be a finite-dimensional vector space. Then a $\mathcal{P}$-coalgebra structure on $V$ is determined by a $\mathbf{k}\left[\Sigma_{2}\right]$ equivariant morphism $\pi: E=\mathcal{P}(2) \rightarrow \operatorname{Coend}(V)(2)$, such that $\hat{\pi}(3)(R)=0$, where $\hat{\pi}: \mathcal{F}(E) \rightarrow$ Coend $(V)$ is the unique operad morphism associated to $\pi$.

Proof. The same proof as Proposition 2.2 works here. Indeed, if $\pi$ is as stated, then the morphism $\hat{\pi}$ must factor through the quotient $\mathcal{P}=\mathcal{F}(E) /(R)$. This is because $\hat{\pi}$ commutes with the $\circ_{i}$ operations and every element in the ideal $(R)$ is a sum of elements that are iterated $\circ_{i}$ products with at least one entry in $R$.

The condition $\hat{\pi}(3)(R)=0$ can be expressed as $[\pi, \pi]=0$ (Corollary 4.5).

\subsection{Duality isomorphism}

Let $V$ be a finite-dimensional vector space. Denote by $V^{\#}$ its linear dual $\operatorname{Hom}(V, \mathbf{k})$. Then for each $n \geq 1$, there is a linear isomorphism (see [26, Proposition 2.8])

$$
\zeta^{n}: \operatorname{Coend}(V)(n) \stackrel{\cong}{\operatorname{End}}\left(V^{\#}\right)(n)
$$

given by $\zeta^{n}(f)=f^{\#}$, where

$$
f^{\#}\left(\alpha_{1} \otimes \cdots \otimes \alpha_{n}\right)(a)=\sum\left(\prod_{i=1}^{n} \alpha_{i}\left(f(a)_{i}\right)\right)
$$

for $\alpha_{i} \in V^{\#}$ and $a \in V$. The notations on the right-hand side of the previous line is given by

$$
f(a)=\sum f(a)_{1} \otimes \cdots \otimes f(a)_{n} \in V^{\otimes n}
$$

Theorem 3.2. Let $V$ be a finite-dimensional vector space. Then the maps $\zeta^{n}(n \geq 1)$ assemble

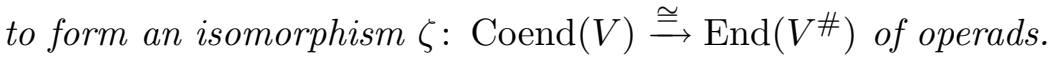

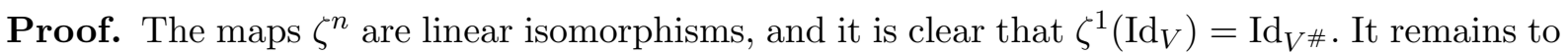
check that they are compatible with the operad structure maps $\gamma$ and that $\zeta^{n}$ is $\Sigma_{n}$-equivariant. For $f \in \operatorname{Coend}(V)(k)$ and $g_{i} \in \operatorname{Coend}(V)\left(n_{i}\right)(1 \leq i \leq k)$, we have

$$
\begin{aligned}
\zeta^{n_{1}+\cdots+n_{k}}\left(\gamma\left(f ; g_{1}, \ldots, g_{k}\right)\right) & =\left(\left(g_{1} \otimes \cdots \otimes g_{k}\right) \circ f\right)^{\#} \\
& =f^{\#} \circ\left(g_{1}^{\#} \otimes \cdots \otimes g_{k}^{\#}\right) \\
& =\gamma\left(\zeta^{k}(f) ; \zeta^{n_{1}}\left(g_{1}\right), \ldots, \zeta^{n_{k}}\left(g_{k}\right)\right)
\end{aligned}
$$

where $\circ$ denotes composition of functions. This shows that $\zeta$ is compatible with the operad structure maps. 
One can see that the map $\zeta^{n}$ is $\Sigma_{n}$-equivariant because the $\Sigma_{n}$-action on $\operatorname{Coend}(V)(n)$ (resp., $\left.\operatorname{End}\left(V^{\#}\right)(n)\right)$ comes from composition with the right (resp., left) $\Sigma_{n}$-action on $V^{\otimes n}$ (resp., $\left.\left(V^{\#}\right)^{\otimes n}\right)$. Indeed, pick $f \in \operatorname{Coend}(V)(n), \sigma \in \Sigma_{n}, \alpha_{i} \in V^{\#}(1 \leq i \leq n)$, and $a \in V$. Then we have

$$
\begin{aligned}
\left(\zeta^{n}(f \cdot \sigma)\right)\left(\alpha_{1} \otimes \cdots \otimes \alpha_{n}\right)(a) & =\sum\left\{\prod_{i=1}^{n} \alpha_{i}\left(f(a)_{\sigma(i)}\right)\right\} \\
& \stackrel{(*)}{=} \sum\left\{\prod_{i=1}^{n} \alpha_{\sigma^{-1}(i)}\left(f(a)_{i}\right)\right\} \\
& =\left(\zeta^{n}(f)\right)\left(\alpha_{\sigma^{-1}(1)} \otimes \cdots \otimes \alpha_{\sigma^{-1}(n)}\right)(a) \\
& =\left(\zeta^{n}(f) \cdot \sigma\right)\left(\alpha_{1} \otimes \cdots \otimes \alpha_{n}\right)(a)
\end{aligned}
$$

The equality $(*)$ comes from the fact that the two sets of elements in $\mathbf{k},\left\{\alpha_{i}\left(f(a)_{\sigma(i)}\right)\right\}_{1 \leq i \leq n}$ and $\left\{\alpha_{\sigma^{-1}(i)}\left(f(a)_{i}\right)\right\}_{1 \leq i \leq n}$, are equal. This shows that $\zeta^{n}$ is $\Sigma_{n}$-equivariant.

This result leads to the following alternative characterizations of a $\mathcal{P}$-coalgebra structure.

Theorem 3.3. Let $\mathcal{P}=\mathcal{P}(\mathbf{k}, E, R)$ be a finitely generated quadratic operad, and let $V$ be a finitedimensional vector space. Then the following three sets are in bijection with each other:

(1) The set of $\mathcal{P}$-coalgebra structures on $V$.

(2) The set of $\mathbf{k}\left[\Sigma_{2}\right]$-equivariant morphisms $\pi: E \rightarrow \operatorname{Coend}(V)(2)$ such that $\hat{\pi}(3)(r)=0$ for $r \in R$.

(3) The set of degree 1 derivations $d \in \operatorname{Der}^{1}\left(\mathcal{F}_{\mathcal{P}^{!}}^{g r}(V)\right)$ that satisfy $d^{2}=0$.

Proof. The bijection between (1) and (2) is Proposition 3.1. By Theorem 2.6, a derivation $d$ as stated corresponds to a $\mathcal{P}$-algebra structure on $V^{\#}$, i.e., an operad morphism $\varphi: \mathcal{P} \rightarrow \operatorname{End}\left(V^{\#}\right)$. Therefore, it follows from Theorem 3.2 that the composition $\zeta^{-1} \varphi: \mathcal{P} \rightarrow \operatorname{Coend}(V)$ is also a morphism of operads. This is by definition a $\mathcal{P}$-coalgebra structure on $V$. The argument can be reversed to prove the converse, thereby giving a bijection between (1) and (3).

\section{Cohomology of $\mathcal{P}$-coalgebras}

In this section, we give another characterization of a $\mathcal{P}$-coalgebra structure in terms of a graded Lie bracket and define cohomology of $\mathcal{P}$-coalgebras.

Throughout this section, let $V$ be a finite-dimensional vector space, and let $\mathcal{P}=\mathcal{P}(\mathbf{k}, E, R)$ be a finitely generated quadratic operad.

\subsection{The graded Lie algebra $\bar{L}_{\mathcal{P}}(V)$}

Define the vector spaces

$$
\begin{array}{rlrl}
\bar{L}_{\mathcal{P}}^{n}(V) & =\mathcal{P}^{!}(n+1) \otimes_{\Sigma_{n+1}} \overline{\operatorname{Coend}}(V)(n+1), & & n \geq 0 \\
\bar{C}_{\mathcal{P}}^{n}(V) & =\operatorname{Hom}\left(V, \mathcal{P}^{!}(n) \otimes \Sigma_{n}\left(V^{\otimes n} \otimes \mathbf{s g n}_{n}\right)\right), & & n \geq 1 \\
& =\operatorname{Hom}\left(V, \mathcal{F}_{\mathcal{P} !}^{g r_{n}}(V)\right) &
\end{array}
$$

Here $\overline{\operatorname{Coend}}(V)(n)=\operatorname{Hom}\left(V, V^{\otimes n}\right) \otimes \mathbf{s g n}_{n}$, which is the same as $\operatorname{Hom}\left(V, V^{\otimes n}\right)$ as a vector space and has the natural left $\Sigma_{n}$-action. 
Theorem 4.1. For each $n \geq 1$, there is an isomorphism $\bar{\Gamma}: \bar{L}_{\mathcal{P}}^{n-1}(V) \cong{ }_{\mathcal{P}}^{n}(V)$ of vector spaces.

Proof. The isomorphism $\bar{\Gamma}$ is the composition of the following isomorphisms:

$$
\begin{aligned}
\bar{L}_{\mathcal{P}}^{n-1}(V) & =\left\{\mathcal{P}^{!}(n) \otimes\left(\operatorname{Hom}\left(V, V^{\otimes n}\right) \otimes \mathbf{s g n}_{n}\right)\right\}_{\Sigma_{n}} \\
& \stackrel{(1)}{\cong}\left\{\mathcal{P}^{!}(n) \otimes\left(\operatorname{Hom}\left(V, V^{\otimes n}\right) \otimes \mathbf{s g n}_{n}\right)\right\}^{\Sigma_{n}} \\
& \stackrel{(2)}{\cong} \operatorname{Hom}_{\mathbf{k}\left[\Sigma_{n}\right]}\left(\left(\mathcal{P}^{!}\right)^{\#}(n), \operatorname{Hom}\left(V, V^{\otimes n}\right) \otimes \mathbf{s g n}_{n}\right) \\
& \cong \operatorname{Hom}\left(V, \operatorname{Hom}_{\mathbf{k}\left[\Sigma_{n}\right]}\left(\left(\mathcal{P}^{!}\right)^{\#}(n), V^{\otimes n} \otimes \mathbf{s g n}_{n}\right)\right) \\
& \stackrel{(3)}{\cong} \operatorname{Hom}\left(V,\left(\mathcal{P}^{!}(n) \otimes\left(V^{\otimes n} \otimes \mathbf{s g n}_{n}\right)\right)^{\Sigma_{n}}\right) \\
& \stackrel{(4)}{\cong} \operatorname{Hom}\left(V, \mathcal{P}^{!}(n) \otimes_{\Sigma_{n}}\left(V^{\otimes n} \otimes \mathbf{s g n}_{n}\right)\right)=\bar{C}_{\mathcal{P}}^{n}(V)
\end{aligned}
$$

The isomorphisms (1) and (4) use the fact that $\left|\Sigma_{n}\right|=n$ ! is invertible in $\mathbf{k}$, which implies that there is a canonical isomorphism $X_{\Sigma_{n}} \cong X^{\Sigma_{n}}$ whenever $X$ is a right $\Sigma_{n}$-module (see, e.g., [23, (3.60)]). The isomorphisms (2) and (3) rely on the fact that $\mathcal{P}^{!}(n)$ is finite-dimensional.

Tracing through the various isomorphisms above, $\bar{\Gamma}$ can be described more explicitly as

$$
\bar{\Gamma}(\mu \otimes \phi)(v)=\frac{1}{n !} \sum_{\sigma \in \Sigma_{n}} \epsilon(\sigma) \mu \sigma \otimes\left\langle\sigma^{-1} \phi, v\right\rangle
$$

where $\mu \in \mathcal{P}^{!}(n), \phi \in \overline{\operatorname{Coend}}(V)(n)$, and $v \in V$.

Using the $\circ_{i}$ operations on the operad $\mathcal{P}^{!}$and the non- $\Sigma$ operad $\overline{\operatorname{Coend}}(V)$, one defines the operations $\circ$ and $[-,-]$ on $\bar{L}_{\mathcal{P}}^{*}(V)$ exactly as in $(2.3)$.

Proposition 4.2. $\left(\bar{L}_{\mathcal{P}}^{*}(V),[-,-]\right)$ is a graded Lie algebra.

Proof. The same argument as in [3, Proposition 2.4.4] shows that the operations $\circ$ and $[-,-]$ are well defined on $\bar{L}_{\mathcal{P}}^{*}(V)$. Since $\mathcal{P}^{!} \otimes \overline{\operatorname{Coend}}(V)$ is a non- $\Sigma$ operad, the result follows from $[8,(3)]$.

\subsection{The graded Lie algebra $\bar{C}_{\mathcal{P}}^{*}(V)$}

Define the operations $\circ$ and $[-,-]$ on $\bar{C}_{\mathcal{P}}^{*}(V)$ via $\bar{\Gamma}$. Namely, define

$$
f \circ g:=\bar{\Gamma}\left(\bar{\Gamma}^{-1}(f) \circ \bar{\Gamma}^{-1}(g)\right), \quad[f, g]:=\bar{\Gamma}\left(\left[\bar{\Gamma}^{-1}(f), \bar{\Gamma}^{-1}(g)\right]\right), \quad f, g \in \bar{C}_{\mathcal{P}}^{*}(V)
$$

The following result is an immediate consequence of Theorem 4.1 and Proposition 4.2.

Corollary 4.3. $\left(\bar{C}_{\mathcal{P}}^{*}(V),[-,-]\right)$ is a graded Lie algebra of degree -1 .

There is another graded Lie bracket on $\bar{C}_{\mathcal{P}}^{*}(V)$ defined in terms of differential derivations using the following result.

Proposition 4.4. There is an isomorphism $\omega$ : $\operatorname{Der}^{n-1}\left(\mathcal{F}_{\mathcal{P} !}^{g r}(V)\right) \stackrel{\cong}{\longrightarrow} \bar{C}_{\mathcal{P}}^{n}(V)$ of vector spaces. 
Proof. Applying Proposition 2.5 to $V$ instead of $V^{\#}$, we obtain the first isomorphism:

$$
\begin{aligned}
\operatorname{Der}^{n-1}\left(\mathcal{F}_{\mathcal{P}^{!}}^{g r}(V)\right) & \stackrel{\omega}{\cong} \operatorname{Hom}\left(\left(\mathcal{P}^{!}\right)^{\#}(n) \otimes \Sigma_{n}\left(V^{\#}\right)^{\otimes n}, V^{\#}\right) \\
& \cong \operatorname{Hom}\left(V, \mathcal{P}^{!}(n) \otimes \Sigma_{n}\left(V^{\otimes n} \otimes \operatorname{sgn}_{n}\right)\right)=\bar{C}_{\mathcal{P}}^{n}(V)
\end{aligned}
$$

The second isomorphism is simply dualization.

The natural graded Lie algebra structure on the space $\operatorname{Der}\left(\mathcal{F}_{\mathcal{P} !}^{g r}(V)\right)$ of derivations induces a graded Lie bracket of degree -1 on $\bar{C}_{\mathcal{P}}^{*}(V)$ via $\omega$ (Proposition 4.4). Exactly as in the case of $\mathcal{P}$-algebra [3, p. 221], this graded Lie bracket is equal to the one defined before (Corollary 4.3).

\subsection{Square-zero characterization of $\mathcal{P}$-coalgebras}

Using the graded Lie bracket on $\bar{C}_{\mathcal{P}}^{*}(V)$, we can give another characterization of a $\mathcal{P}$-coalgebra structure, adding to the list in Theorem 3.3.

Corollary 4.5. There is a one-to-one correspondence between the $\mathcal{P}$-coalgebra structures on $V$ and elements $\varphi \in \bar{C}_{\mathcal{P}}^{2}(V)$ satisfying $[\varphi, \varphi]=0$.

Proof. As in [3, Corollary 2.4.2], under the isomorphism $\omega$ in Proposition 4.4, an element $d \in \operatorname{Der}^{1}\left(\mathcal{F}_{\mathcal{P} !}^{g r}(V)\right)$ corresponds to an element $\varphi \in \bar{C}_{\mathcal{P}}^{2}(V)$ such that $[\varphi, \varphi]=2 d^{2}$. The result now follows from Theorem 3.3.

\subsection{Coboundary in $\bar{C}_{\mathcal{P}}^{*}(V)$}

Now let $V$ be a finite-dimensional $\mathcal{P}$-coalgebra with structural morphism $\pi \in \bar{C}_{\mathcal{P}}^{2}(V)$, i.e., $[\pi, \pi]=0$. Following Balavoine [3], define a map $\bar{\delta}_{\pi}^{n}: \bar{C}_{\mathcal{P}}^{n}(V) \rightarrow \bar{C}_{\mathcal{P}}^{n+1}(V)$ by setting

$$
\bar{\delta}_{\pi}^{n}(f)=-\frac{n+1}{2}[f, \pi]
$$

for $f \in \bar{C}_{\mathcal{P}}^{n}(V)$. Note that this has the exact same formula as the differential in $C_{\mathcal{P}}^{*}(V)(2.5)$.

Corollary 4.6. The map $\bar{\delta}_{\pi}$ is a differential on $\bar{C}_{\mathcal{P}}^{*}(V)$. In particular, $\left(\bar{C}_{\mathcal{P}}^{*}(V), \bar{\delta}_{\pi},[-,-]\right)$ is a differential graded Lie algebra.

Proof. The map $\bar{\delta}_{\pi}^{n}=-\frac{n+1}{2}[-, \pi]$ is a differential because $[-,-]$ is a graded Lie bracket and $[\pi, \pi]=0$.

\subsection{Cohomology of $\mathcal{P}$-coalgebras}

The cohomology of the cochain complex $\left(\bar{C}_{\mathcal{P}}^{*}(V), \bar{\delta}_{\pi}\right)$ is denoted by $\bar{H}_{\mathcal{P}}^{n}(V)$ or $\bar{H}_{\mathcal{P}}^{n}(V, \pi)$ and is called the cohomology of $V$ with coefficients in itself.

Essentially the same discussion as in [3, Section 4] also applies here, showing that the differential graded Lie algebra $\left(\bar{C}_{\mathcal{P}}^{*}(V), \bar{\delta}_{\pi},[-,-]\right)$ controls the deformations of the $\mathcal{P}$-coalgebra $(V, \pi)$.

The following result is an immediate consequence of Corollary 4.6.

Corollary 4.7. The graded vector space $\bar{H}_{\mathcal{P}}^{*}(V)$ inherits the structure of a graded Lie algebra from $\bar{C}_{\mathcal{P}}^{*}(V)$. 
Example 4.8 (Hochschild coalgebra cohomology). Let $V$ be a finite-dimensional As-coalgebra (i.e., a finite-dimensional coassociative coalgebra) with comultiplication $\Delta: V \rightarrow V^{\otimes 2}$. Using the fact $[9] \mathbf{A s}=\mathbf{A} \mathbf{s}^{!}=\left\{\mathbf{k}\left[\Sigma_{n}\right]\right\}$, we deduce that $\bar{C}_{\mathbf{A} \mathbf{s}}^{n}(V)=\operatorname{Hom}\left(V, V^{\otimes n}\right)$ and $\bar{H}_{\mathbf{A s}}^{n}(V)=$ $H_{c}^{n}(V, V) \quad(n \geq 2)$, where $H_{c}^{*}(V, V)$ denotes the Hochschild coalgebra cohomology of $V$ with self-coefficients $[12,26]$. The last equality comes from the fact (see Theorems 6.1 and 6.2) that $\frac{2}{n+1} \bar{\delta}_{\pi}^{n}=b_{c}^{n}$, where $b_{c}$ is the Hochschild coalgebra coboundary on the cochain complex $\left\{\operatorname{Hom}\left(V, V^{\otimes n}\right)\right\}$. More precisely, it is defined as the alternating sum

$$
\begin{aligned}
b_{c}^{n}(f)= & \left(\operatorname{Id}_{V} \otimes f\right) \circ \Delta+(-1)^{n+1}\left(f \otimes \operatorname{Id}_{V}\right) \circ \Delta \\
& +\sum_{i=1}^{n}(-1)^{i}\left(\operatorname{Id}_{V}^{\otimes(i-1)} \otimes \Delta \otimes \operatorname{Id}_{V}^{\otimes(n-i)}\right) \circ f
\end{aligned}
$$

for $f \in \operatorname{Hom}\left(V, V^{\otimes n}\right)$, where $\circ$ denotes composition of functions.

Using (2.3), one observes that the graded Lie bracket on $\bar{C}_{\mathbf{A s}}^{*}(V)$ (and hence also on $\bar{H}_{\mathbf{A s}}^{*}(V)$ $\left.=H_{c}^{*}(V, V)\right)$ is given by

$$
\begin{aligned}
{[f, g]=} & \sum_{i=1}^{m}(-1)^{(n-1)(i-1)}\left(\operatorname{Id}_{V}^{\otimes(i-1)} \otimes g \otimes \operatorname{Id}_{V}^{\otimes(m-i)}\right) \circ f \\
& -(-1)^{(m-1)(n-1)} \sum_{j=1}^{n}(-1)^{(m-1)(j-1)}\left(\operatorname{Id}_{V}^{\otimes(j-1)} \otimes f \otimes \operatorname{Id}_{V}^{\otimes(n-j)}\right) \circ g
\end{aligned}
$$

for $f \in \bar{C}_{\mathbf{A} \mathbf{s}}^{m}(V)$ and $g \in \bar{C}_{\mathbf{A} \mathbf{s}}^{n}(V)$.

\section{Cohomological duality}

Throughout this section, let $V$ be a finite-dimensional vector space, and let $\mathcal{P}=\mathcal{P}(\mathbf{k}, E, R)$ be a finitely generated quadratic operad. Recall that $V^{\#}=\operatorname{Hom}(V, \mathbf{k})$ denotes the linear dual of $V$. The purpose of this section is to show that $\bar{H}_{\mathcal{P}}^{*}(V)$ and $H_{\mathcal{P}}^{*}\left(V^{\#}\right)$ are isomorphic as graded Lie algebras.

\subsection{Cochain level isomorphism}

Consider the map

$$
\xi^{n} \stackrel{\text { def }}{=} \Gamma\left(\operatorname{Id}_{\mathcal{P} !(n)} \otimes \zeta^{n}\right) \bar{\Gamma}^{-1}: \bar{C}_{\mathcal{P}}^{n}(V) \rightarrow C_{\mathcal{P}}^{n}\left(V^{\#}\right)
$$

for $n \geq 1$. It is clear that $\xi^{n}$ is a linear isomorphism, since each of the three maps that define it is an isomorphism (Proposition 2.4, (3.1), and Theorem 4.1).

Using the formulas for $\Gamma(2.4), \bar{\Gamma}(4.1)$, and $\zeta^{n}(3.1)$, one infers that $\xi^{n}$ is, in fact, the dualization isomorphism

$$
\begin{aligned}
\bar{C}_{\mathcal{P}}^{n}(V) & =\operatorname{Hom}\left(V, \mathcal{P}^{!}(n) \otimes_{\Sigma_{n}}\left(V^{\otimes n} \otimes \mathbf{s g n}_{n}\right)\right) \\
& \cong \operatorname{Hom}\left(\left(\mathcal{P}^{!}\right)^{\#}(n) \otimes_{\Sigma_{n}}\left(V^{\#}\right)^{\otimes n}, V^{\#}\right)=C_{\mathcal{P}}^{n}\left(V^{\#}\right)
\end{aligned}
$$

More explicitly, given $\varphi \in \bar{C}_{\mathcal{P}}^{n}(V), \mu \in\left(\mathcal{P}^{!}\right)^{\#}(n), \alpha \in\left(V^{\#}\right)^{\otimes n} \cong\left(V^{\otimes n}\right)^{\#}$, and $x \in V$, we have

$$
\left(\xi^{n} \varphi\right)(\mu \otimes \alpha)(x)=\sum\left\langle\mu, \varphi(x)_{(1)}\right\rangle\left\langle\alpha, \varphi(x)_{(2)}\right\rangle
$$


where

$$
\varphi(x)=\sum \varphi(x)_{(1)} \otimes \varphi(x)_{(2)} \in \mathcal{P}^{!}(n) \otimes_{\Sigma_{n}} V^{\otimes n}
$$

Theorem 5.1. The maps $\xi^{n}$ assemble to form an isomorphism $\xi: \bar{C}_{\mathcal{P}}^{*}(V) \stackrel{\cong}{\longrightarrow} C_{\mathcal{P}}^{*}\left(V^{\#}\right)$ of graded Lie algebras.

Proof. It remains to show that $\xi$ commutes with the graded Lie brackets. It suffices to show that each of the three maps that define $\xi$ commutes with the graded Lie brackets. Both isomorphisms $\Gamma$ and $\bar{\Gamma}^{-1}$ commute with the graded Lie brackets by definition.

On the other hand, the operations $\circ$ and $[-,-]$ on $L_{\mathcal{P}}(V)$ and $\bar{L}_{\mathcal{P}}(V)$ are defined in terms of the $\circ_{i}$ operations in $\mathcal{P}^{!}, \overline{\operatorname{End}}(V)$, and $\overline{\operatorname{Coend}}(V)$. It follows from the fact that the $\circ_{i}$ operations can be written in terms of the operad structure map $\gamma(2.1)$ and Theorem 3.2 that the isomorphism in the middle, $\left(\operatorname{Id}_{\mathcal{P} !(n)} \otimes \zeta^{n}\right)$, commutes with the operation $\circ$ and hence also the graded Lie bracket.

Corollary 5.2. Let $\pi$ be an element in $\bar{C}_{\mathcal{P}}^{2}(V)$. Then $\pi$ defines a $\mathcal{P}$-coalgebra structure on $V$ if and only if $\xi^{2} \pi \in C_{\mathcal{P}}^{2}\left(V^{\#}\right)$ defines a $\mathcal{P}$-algebra structure on $V^{\#}$.

Proof. From Theorem 5.1, it follows that $\xi^{3}([\pi, \pi])=\left[\xi^{2} \pi, \xi^{2} \pi\right]$. The result now follows from the fact that $\xi^{3}$ is an isomorphism, Theorem 2.6, and Corollary 4.5.

Corollary 5.3. Let $(V, \pi)$ be a finite-dimensional $\mathcal{P}$-coalgebra. Then the map

$$
\xi:\left(\bar{C}_{\mathcal{P}}^{*}(V), \bar{\delta}_{\pi},[-,-]\right) \stackrel{\cong}{\longrightarrow}\left(C_{\mathcal{P}}^{*}\left(V^{\#}\right), \delta_{\xi^{2} \pi},[-,-]\right)
$$

is an isomorphism of differential graded Lie algebras.

Proof. In view of Theorem 5.1 and Corollary 5.2, it remains to show that $\xi$ commutes with the differentials. Pick an element $f \in \bar{C}_{\mathcal{P}}^{n}(V)$. Then we have that

$$
\xi^{n+1}\left(\bar{\delta}_{\pi}^{n}(f)\right)=-\frac{n+1}{2} \xi^{n+1}([f, \pi])=-\frac{n+1}{2}\left[\xi^{n} f, \xi^{2} \pi\right]=\delta_{\xi^{2} \pi}^{n}\left(\xi^{n} f\right)
$$

Passing to cohomology, we obtain the following result.

Corollary 5.4. The map $\xi$ induces an isomorphism $\xi^{*}: \bar{H}_{\mathcal{P}}^{*}(V, \pi) \stackrel{\cong}{\longrightarrow} H_{\mathcal{P}}^{*}\left(V^{\#}, \xi^{2} \pi\right)$ of graded Lie algebras.

Example 5.5 (= Example 4.8, continued). Let $V$ be a finite-dimensional coassociative coalgebra (i.e., a finite-dimensional As-coalgebra) with comultiplication $\Delta \in \bar{C}_{\mathbf{A s}}^{2}(V)=\operatorname{Hom}\left(V, V^{\otimes 2}\right)$. Then $\xi^{2} \Delta \in C_{\mathbf{A} \mathbf{s}}^{2}\left(V^{\#}\right)=\operatorname{Hom}\left(\left(V^{\#}\right)^{\otimes 2}, V^{\#}\right)$ is the usual multiplication of the linear dual $V^{\#}$. In this case, Corollary 5.4 says that there is a duality isomorphism $\xi^{*}: H_{c}^{*}(V, V) \stackrel{\cong}{\longrightarrow} H_{h}^{*}\left(V^{\#}, V^{\#}\right)$ of graded Lie algebras, where $H_{h}^{*}\left(V^{\#}, V^{\#}\right)$ denotes the Hochschild cohomology [11] of the associative algebra $V^{\#}$ with self-coefficients. The graded Lie bracket in $H_{c}^{*}(V, V)$ is as described in (4.5). The graded Lie bracket in $H_{h}^{*}\left(V^{\#}, V^{\#}\right)$ is the Gerstenhaber bracket [6]. 


\subsection{Duality isomorphism: From $\mathcal{P}$-algebra to $\mathcal{P}$-coalgebra}

There are also the obvious counterparts of the results above that relate the cohomology of a finitedimensional $\mathcal{P}$-algebra $(V, \pi)$ with that of the finite-dimensional $\mathcal{P}$-coalgebra $\left(V^{\#},\left(\xi^{2}\right)^{-1} \pi\right)$.

Theorem 5.6. Let $(V, \pi)$ be a finite-dimensional $\mathcal{P}$-algebra. Then the map

$$
\xi^{-1}:\left(C_{\mathcal{P}}^{*}(V), \delta_{\pi},[-,-]\right) \stackrel{\cong}{\longrightarrow}\left(\bar{C}_{\mathcal{P}}^{*}\left(V^{\#}\right), \bar{\delta}_{\left(\xi^{2}\right)^{-1} \pi},[-,-]\right)
$$

is an isomorphism of differential graded Lie algebras. Passing to cohomology, it induces an isomorphism

$$
\left(\xi^{-1}\right)^{*}: H_{\mathcal{P}}^{*}(V, \pi) \stackrel{\cong}{\longrightarrow} \bar{H}_{\mathcal{P}}^{*}\left(V^{\#},\left(\xi^{2}\right)^{-1} \pi\right)
$$

of graded Lie algebras.

Since the arguments are essentially the same as the ones given above, we will omit them.

\section{The differential $\bar{\delta}_{\pi}$}

In this section, we give an explicit description of the differential $\bar{\delta}_{\pi}(4.3)$ in $\bar{C}_{\mathcal{P}}^{*}(V)$ for a finitedimensional $\mathcal{P}$-coalgebra $(V, \pi)$, where $\mathcal{P}=\mathcal{P}(\mathbf{k}, E, R)$ is a finitely generated quadratic operad.

\subsection{The component maps $\bar{\delta}_{\pi}^{1, n}$ and $\bar{\delta}_{\pi}^{2, n}$}

Pick elements $f \in \bar{C}_{\mathcal{P}}^{n}(V)$ and $v \in V$. Write

$$
\bar{\Gamma}^{-1}(f)=\sum_{\beta} \nu_{\beta}^{*} \otimes F_{\beta} \in \mathcal{P}^{!}(n) \otimes_{\Sigma_{n}} \overline{\operatorname{Coend}}(V)(n)
$$

and

$$
\bar{\Gamma}^{-1}(\pi)=\sum_{\alpha} \mu_{\alpha}^{*} \otimes \Pi_{\alpha} \in \mathcal{P}^{!}(2) \otimes_{\Sigma_{2}} \overline{\operatorname{Coend}}(V)(2)
$$

Using (4.1), (4.2), (4.3), and the definition of the graded Lie bracket in $\bar{L}_{\mathcal{P}}(V)$ (2.3), one infers that

$$
\bar{\delta}_{\pi}^{n}(f)(v)=\bar{\delta}_{\pi}^{1, n}(f)(v)+\bar{\delta}_{\pi}^{2, n}(f)(v)
$$

where

$$
\frac{2}{n+1} \bar{\delta}_{\pi}^{1, n}(f)(v)=\sum_{i=1}^{n}(-1)^{i} \sum_{\alpha, \beta} \frac{1}{(n+1) !} \sum_{\tau_{i} \in \Sigma_{n+1}} \epsilon\left(\tau_{i}\right)\left(\nu_{\beta}^{*} \circ_{i} \mu_{\alpha}^{*}\right) \tau_{i} \otimes\left\langle\tau_{i}^{-1}\left(F_{\beta} \circ_{i} \Pi_{\alpha}\right), v\right\rangle
$$

and

$$
\frac{2}{n+1} \bar{\delta}_{\pi}^{2, n}(f)(v)=\sum_{i=1}^{2}(-1)^{(n+1) i} \sum_{\alpha, \beta} \frac{1}{(n+1) !} \sum_{\tau_{i} \in \Sigma_{n+1}} \epsilon\left(\tau_{i}\right)\left(\mu_{\alpha}^{*} \circ_{i} \nu_{\beta}^{*}\right) \tau_{i} \otimes\left\langle\tau_{i}^{-1}\left(\Pi_{\alpha} \circ_{i} F_{\beta}\right), v\right\rangle
$$

In view of (6.1), in order to understand the differential $\bar{\delta}_{\pi}^{n}$, it suffices to describe the component $\operatorname{maps} \bar{\delta}_{\pi}^{1, n}$ and $\bar{\delta}_{\pi}^{2, n}$. 


\subsection{Explicit formula for $\bar{\delta}_{\pi}^{1, n}$}

In order to describe $\bar{\delta}_{\pi}^{1, n}(f)(v)$ more explicitly using $f$ itself, we use the notations

$$
\begin{aligned}
f(v) & =\sum_{(z)} z_{(1)} \otimes z_{(2)} \in \mathcal{P}^{!}(n) \otimes \Sigma_{n}\left(V^{\otimes n} \otimes \mathbf{s g n}_{n}\right) \\
\Pi_{\alpha}^{j} & =\operatorname{Id}_{V}^{\otimes(j-1)} \otimes \Pi_{\alpha} \otimes \operatorname{Id}_{V}^{\otimes(n-j)} \quad(1 \leq j \leq n) \\
\sum^{\prime} & =\sum_{\alpha} \sum_{(z)} \sum_{j=1}^{n}(-1)^{j} \sum_{\sigma_{j} \in \Sigma_{n+1}}
\end{aligned}
$$

The following result is the $\mathcal{P}$-coalgebra analogue of [2, Theorem 3.2.3], and it corresponds to the term

$$
\sum_{i=1}^{n}(-1)^{i}\left(\operatorname{Id}_{V}^{\otimes(i-1)} \otimes \Delta \otimes \operatorname{Id}_{V}^{\otimes(n-i)}\right) \circ f
$$

in (4.4).

Theorem 6.1. We have that

$$
\bar{\delta}_{\pi}^{1, n}(f)(v)=\frac{1}{2 n !} \sum^{\prime}\left(z_{(1)} \circ_{j} \mu_{\alpha}^{*}\right) \sigma_{j} \otimes \epsilon\left(\sigma_{j}\right) \sigma_{j}^{-1} \Pi_{\alpha}^{j}\left(z_{(2)}\right)
$$

where the notations are as in (6.3).

The proof will be given below. Notice that the second component in the sum in Theorem 6.1 has an alternative description as follows. If $z_{(2)}=x_{1} \otimes \cdots \otimes x_{n} \in V^{\otimes n}$, then $\Pi_{\alpha}^{j}\left(z_{(2)}\right) \in V^{\otimes(n+1)}$ has the form

$$
\begin{aligned}
\Pi_{\alpha}^{j}\left(z_{(2)}\right) & =\left(\operatorname{Id}_{V}^{\otimes(j-1)} \otimes \Pi_{\alpha} \otimes \operatorname{Id}_{V}^{\otimes(n-j)}\right)\left(x_{1} \otimes \cdots \otimes x_{n}\right) \\
& =x_{1} \otimes \cdots \otimes x_{j-1} \otimes \Pi_{\alpha}\left(x_{j}\right) \otimes x_{j+1} \otimes \cdots \otimes x_{n} \\
& =: \sum y_{1} \otimes \cdots \otimes y_{n+1}
\end{aligned}
$$

It follows that

$$
\epsilon\left(\sigma_{j}\right) \sigma_{j}^{-1} \Pi_{\alpha}^{j}\left(z_{(2)}\right)=\sum y_{\sigma_{j}(1)} \otimes \cdots \otimes y_{\sigma_{j}(n+1)}
$$

\subsection{Explicit formula for $\bar{\delta}_{\pi}^{2, n}$}

To deal with $\bar{\delta}_{\pi}^{2, n}(f)(v)$, we use the notations

$$
\begin{aligned}
\Pi_{\alpha}(v) & =\sum_{(w)} w_{(1)} \otimes w_{(2)} \in V^{\otimes 2} \\
f\left(w_{(i)}\right) & =\sum_{\left(f\left(w_{(i)}\right)\right)} f\left(w_{(i)}\right)_{(1)} \otimes f\left(w_{(i)}\right)_{(2)}, \quad i=1,2 \\
\sum^{i} & =\sum_{\alpha} \sum_{(w)} \sum_{\left(f\left(w_{(i)}\right)\right)} \sum_{\sigma \in \Sigma_{n+1}}, \quad i=1,2
\end{aligned}
$$


The following result is the $\mathcal{P}$-coalgebra analogue of [2, Theorem 3.2.4], and it corresponds to the sum

$$
\left(\operatorname{Id}_{V} \otimes f\right) \circ \Delta+(-1)^{n+1}\left(f \otimes \operatorname{Id}_{V}\right) \circ \Delta
$$

in (4.4).

Theorem 6.2. We have that

$$
\begin{aligned}
\bar{\delta}_{\pi}^{2, n}(f)(v)= & \frac{1}{2 n !} \sum^{2} \epsilon(\sigma)\left(\mu_{\alpha}^{*} \circ_{2} f\left(w_{(2)}\right)_{(1)}\right) \sigma \otimes \sigma^{-1}\left(w_{(1)} \otimes f\left(w_{(2)}\right)_{(2)}\right) \\
& +(-1)^{n+1} \frac{1}{2 n !} \sum^{1} \epsilon(\sigma)\left(\mu_{\alpha}^{*} \circ_{1} f\left(w_{(1)}\right)_{(1)}\right) \sigma \otimes \sigma^{-1}\left(f\left(w_{(1)}\right)_{(2)} \otimes w_{(2)}\right)
\end{aligned}
$$

where the notations are as in (6.4).

Proof of Theorem 6.1. We follow the notations and parts of the arguments in [2, 3.2.2]. For a fixed element $\tau \in \Sigma_{n}$, we set

$$
\tau_{i}=\left(\tau \circ_{i} 1_{\Sigma_{2}}\right) \tau_{i}^{\prime} \in \Sigma_{n+1}
$$

for $\tau_{i}^{\prime} \in \Sigma_{n+1}$. According to $[3,(7)]$, the signature of $\tau_{i}$ is given by

$$
\epsilon\left(\tau_{i}\right)=(-1)^{i-\tau^{-1}(i)} \epsilon(\tau) \epsilon\left(\tau_{i}^{\prime}\right)
$$

It follows that

$$
\begin{aligned}
(-1)^{i} \epsilon\left(\tau_{i}\right)\left(\nu_{\beta}^{*} \circ_{i} \mu_{\alpha}^{*}\right) \tau_{i} & =(-1)^{\tau^{-1}(i)} \epsilon(\tau) \epsilon\left(\tau_{i}^{\prime}\right)\left(\nu_{\beta}^{*} \circ_{i} \mu_{\alpha}^{*}\right)\left(\tau \circ_{i} 1_{\Sigma_{2}}\right) \tau_{i}^{\prime} \\
& =(-1)^{\tau^{-1}(i)} \epsilon(\tau) \epsilon\left(\tau_{i}^{\prime}\right)\left(\nu_{\beta}^{*} \tau \circ_{\tau^{-1}(i)} \mu_{\alpha}^{*}\right) \tau_{i}^{\prime}
\end{aligned}
$$

Likewise, we have

$$
\tau_{i}^{-1}\left(F_{\beta} \circ_{i} \Pi_{\alpha}\right)=\left(\tau_{i}^{\prime}\right)^{-1}\left(\tau^{-1} F_{\beta} \circ_{\tau^{-1}(i)} \Pi_{\alpha}\right)
$$

Now set $j=\tau^{-1}(i)$ and $\sigma_{j}=\tau_{i}^{\prime}$. Substituting (6.5) and (6.6) back into (6.2), it follows that

$$
2 n ! \bar{\delta}_{\pi}^{1, n}(f)(v)=\sum_{j=1}^{n}(-1)^{j} \sum_{\sigma_{j} \in \Sigma_{n+1}} \epsilon(\tau) \epsilon\left(\sigma_{j}\right) \sum_{\alpha, \beta}\left(\nu_{\beta}^{*} \tau \circ_{j} \mu_{\alpha}^{*}\right) \sigma_{j} \otimes\left\langle\sigma_{j}^{-1}\left(\tau^{-1} F_{\beta} \circ_{j} \Pi_{\alpha}\right), v\right\rangle
$$

Since (6.7) holds for any $\tau \in \Sigma_{n}$, we can average it over $\Sigma_{n}$, which gives rise to

$$
2 n ! \bar{\delta}_{\pi}^{1, n}(f)(v)=\sum_{\substack{1 \leq j \leq n \\ \sigma_{j} \in \Sigma_{n+1} \\ \alpha}}(-1)^{j} \epsilon\left(\sigma_{j}\right) \sum_{\substack{\tau \in \Sigma_{n} \\ \beta}}\left[\frac{\epsilon(\tau) \nu_{\beta}^{*} \tau}{n !} \circ_{j} \mu_{\alpha}^{*}\right] \sigma_{j} \otimes\left\langle\sigma_{j}^{-1}\left(\tau^{-1} F_{\beta} \circ_{j} \Pi_{\alpha}\right), v\right\rangle
$$

Since the second $\circ_{j}$ is in $\overline{\operatorname{Coend}}(V)$, the last component in (6.8) can be rewritten as

$$
\left\langle\sigma_{j}^{-1}\left(\tau^{-1} F_{\beta} \circ_{j} \Pi_{\alpha}\right), v\right\rangle=\sigma_{j}^{-1} \Pi_{\alpha}^{j}\left\langle\tau^{-1} F_{\beta}, v\right\rangle
$$


To finish the proof, note that by using the explicit formula for $\bar{\Gamma}(4.1)$, we can also write $f(v)$ as

$$
\begin{aligned}
f(v) & =\bar{\Gamma}\left(\bar{\Gamma}^{-1}(f)\right)(v)=\sum_{\beta} \bar{\Gamma}\left(\nu_{\beta}^{*} \otimes F_{\beta}\right)(v) \\
& =\sum_{\beta} \sum_{\tau \in \Sigma_{n}} \frac{\epsilon(\tau) \nu_{\beta}^{*} \tau}{n !} \otimes\left\langle\tau^{-1} F_{\beta}, v\right\rangle=\sum_{(z)} z_{(1)} \otimes z_{(2)}
\end{aligned}
$$

The proof of Theorem 6.1 now finishes by combining (6.8), (6.9), and (6.10).

Theorem 6.2 can be proved by modifying the proof of Theorem 6.1 slightly.

\subsection{Example: $\bar{\delta}_{\pi}^{1}$}

As an example, we compute $\bar{\delta}_{\pi}^{1}(f)(v)$ explicitly using Theorems 6.1 and 6.2 . Given $f \in \bar{C}_{\mathcal{P}}^{1}(V)$ and $v \in V$, we have that $f(v)=\sum z_{(1)} \otimes z_{(2)}$, which can be considered as an element in $V$ because $z_{(1)} \in \mathcal{P}^{!}(1)=\mathbf{k}, z_{(2)} \in V$, and $\otimes_{\mathbf{k}\left[\Sigma_{1}\right]}=\otimes_{\mathbf{k}}$. Since $1 \circ_{1} \mu_{\alpha}^{*}=\mu_{\alpha}^{*}$, it follows that

$$
\begin{aligned}
\bar{\delta}_{\pi}^{1,1}(f)(v) & =-\frac{1}{2} \sum_{\substack{\alpha,(z) \\
\sigma \in \Sigma_{2}}}\left(z_{(1)} \circ_{1} \mu_{\alpha}^{*}\right) \sigma \otimes \epsilon(\sigma) \sigma^{-1} \Pi_{\alpha}\left(z_{(2)}\right) \\
& =-\frac{1}{2} \sum_{\alpha} \sum_{\sigma \in \Sigma_{2}} \mu_{\alpha}^{*} \sigma \otimes \epsilon(\sigma)\left\langle\sigma^{-1} \Pi_{\alpha}, f(v)\right\rangle=-\pi(f(v))
\end{aligned}
$$

The last equality follows from the formula for $\bar{\Gamma}(4.1)$.

Likewise, since $\mu_{\alpha}^{*} \circ_{i} 1=\mu_{\alpha}^{*}$ for $i \in\{1,2\}$, a similar analysis as above shows that

$$
\bar{\delta}_{\pi}^{2,1}(f)(v)=\left(\operatorname{Id}_{\mathcal{P} !(2)} \otimes \operatorname{Id}_{V} \otimes f\right)(\pi(v))+\left(\operatorname{Id}_{\mathcal{P} !(2)} \otimes f \otimes \operatorname{Id}_{V}\right)(\pi(v))
$$

Combining (6.11) and (6.12), we have that

$$
\bar{\delta}_{\pi}^{1}(f)=\left(\operatorname{Id}_{\mathcal{P} !(2)} \otimes \operatorname{Id}_{V} \otimes f\right) \circ \pi-\pi \circ f+\left(\operatorname{Id}_{\mathcal{P} !(2)} \otimes f \otimes \operatorname{Id}_{V}\right) \circ \pi
$$

in which $\circ$ denotes composition of functions.

\section{5 $\bar{H}_{\mathcal{P}}^{1}(V)$ as coderivations}

Recall that for a coassociative coalgebra $(C, \Delta)$, a coderivation on $C$ is a linear map $f: C \rightarrow C$ such that

$$
\Delta(f(x))=\sum_{(x)} x_{(1)} \otimes f\left(x_{(2)}\right)+f\left(x_{(1)}\right) \otimes x_{(2)}
$$

for $x \in C$, where $\Delta(x)=\sum_{(x)} x_{(1)} \otimes x_{(2)} \in C \otimes C$. The condition (6.14) can also be stated in the element-free form as

$$
\Delta \circ f=\left(\operatorname{Id}_{C} \otimes f+f \otimes \operatorname{Id}_{C}\right) \circ \Delta
$$


We generalize this to $\mathcal{P}$-coalgebras. For a finite-dimensional $\mathcal{P}$-coalgebra $(V, \pi)$, we define a coderivation on $(V, \pi)$ to be a linear map $f: V \rightarrow V$ such that

$$
\pi \circ f=\left(\operatorname{Id}_{\mathcal{P} !(2)} \otimes \operatorname{Id}_{V} \otimes f+\operatorname{Id}_{\mathcal{P} !(2)} \otimes f \otimes \operatorname{Id}_{V}\right) \circ \pi
$$

Here $\circ$ denotes composition of functions, and we regard $\pi$ as an element of

$$
\bar{C}_{\mathcal{P}}^{2}(V)=\operatorname{Hom}\left(V, \mathcal{P}^{!}(2) \otimes_{\Sigma_{2}}\left(V^{\otimes 2} \otimes \operatorname{sgn}_{2}\right)\right)
$$

Denote by $\operatorname{Coder}_{\mathcal{P}}(V)$ the vector space of all coderivations on $(V, \pi)$.

The formula $(6.13)$ for $\bar{\delta}_{\pi}^{1}(f)$ shows that $f \in \bar{C}_{\mathcal{P}}^{1}(V)$ is annihilated by $\bar{\delta}_{\pi}^{1}$ if and only if $f$ is a coderivation on $(V, \pi)$. This leads to the following result.

Corollary 6.3. There is an equality $\bar{H}_{\mathcal{P}}^{1}(V)=\operatorname{Coder}_{\mathcal{P}}(V)$ of vector spaces.

\section{Acknowledgement}

The first author is supported by NBHM Post doctoral fellowship.

\section{References}

[1] M. Aguiar and J.-L. Loday. Quadri-algebras. J. Pure Appl. Algebra, 191 (2004), 205-221.

[2] D. Balavoine. Homology and cohomology with coefficients, of an algebra over a quadratic operad. J. Pure Appl. Algebra, 132 (1998), 221-258.

[3] D. Balavoine. Deformations of algebras over a quadratic operad. In "Operads: Proceedings of Renaissance Conferences" (Hartford, CT/Luminy, 1995). J.-L. Loday, J. D. Stasheff, and A. A. Voronov, Eds. Contemporary Mathematics 202, American Mathematical Society, Providence, RI, 1997, 207234.

[4] H. Cartan and S. Eilenberg. Homological Algebra. Princeton University Press, Princeton, 1956.

[5] A. Frabetti. Dialgebra (co)homology with coefficients. In "Dialgebras and Related Operads". J.M. Morel, F. Takens, and B. Teissier, Eds. Lecture Notes in Mathematics 1763, Springer-Verlag, Berlin, 2001, 67-103.

[6] M. Gerstenhaber. The cohomology structure of an associative ring. Ann. Math., 78 (1963), 267-288.

[7] M. Gerstenhaber. On the deformation of rings and algebras. Ann. Math., 79 (1964), 59-103.

[8] M. Gerstenhaber and A. A. Voronov. Homotopy $G$-algebras and moduli space operad. Int. Math. Res. Notices, 1995 (1995), 141-153.

[9] V. Ginzburg and M. M. Kapranov. Koszul duality for operads. Duke Math. J., 76 (1994), 203-272.

[10] D. K. Harrison. Commutative algebras and cohomology. Trans. Amer. Math. Soc., 104 (1962), 191-204.

[11] G. Hochschild. On the cohomology groups of an associative algebra. Ann. Math., 46 (1945), 58-67.

[12] D. W. Jonah. Cohomology of coalgebras. Memoirs of the American Mathematical Society, No. 82, American Mathematical Society, Providence, R.I., 1968.

[13] Ph. Leroux. Ennea-algebras. J. Algebra, 281 (2004), 287-302.

[14] J.-L. Loday. Cyclic homology. Grundlehren der mathematischen Wissenschaften 301, SpringerVerlag, Berlin, 1992.

[15] J.-L. Loday. Une version non commutative des algèbres de Lie: les algèbres de Leibniz. Ens. Math., 39 (1993), 269-293. 
[16] J.-L. Loday. Cup-product for Leibniz cohomology and dual Leibniz algebras. Math. Scand., 77 (1995), 189-196.

[17] J.-L. Loday. Dialgebras. In "Dialgebras and Related Operads". J.-M. Morel, F. Takens, and B. Teissier, Eds. Lecture Notes in Mathematics 1763, Springer-Verlag, Berlin, 2001, 7-66.

[18] J.-L. Loday and T. Pirashvili. Universal enveloping algebras of Leibniz algebras and (co)homology. Math. Ann., 296 (1993), 139-158.

[19] J.-L. Loday and M. Ronco. Trialgebras and families of polytopes. In "Homotopy Theory: Relations with Algebraic Geometry, Group Cohomology, and Algebraic K-Theory". P. Goerss and S. Priddy, Eds. Contemporary Mathematics 346, American Mathematical Society, Providence, RI, 2004, 369398.

[20] A. Majumdar and G. Mukherjee. Deformation theory of dialgebras. K-theory, 27 (2002), 33-60.

[21] A. Majumdar and G. Mukherjee. Dialgebra cohomology as a G-algebra. Trans. Amer. Math. Soc., 356 (2004), 2443-2457.

[22] M. Markl. Models for operads. Comm. Alg., 24 (1996), 1471-1500.

[23] M. Markl, S. Shnider, and J. Stasheff. Operads in Algebra, Topology and Physics. Mathematical Surveys and Monographs 96, American Mathematical Society, Providence, RI, 2002.

[24] J. P. May. The Geometry of Iterated Loop Spaces. Lectures Notes in Mathematics 271, SpringerVerlag, Berlin, 1972.

[25] J. P. May. Definitions: Operads, algebras and modules. In "Operads: Proceedings of Renaissance Conferences" (Hartford, CT/Luminy, 1995). J.-L. Loday, J. D. Stasheff, and A. A. Voronov, Eds. Contemporary Mathematics 202, American Mathematical Society, Providence, RI, 1997, 1-7.

[26] B. Parshall and J. P. Wang. On bialgebra cohomology. Algebra, groups and geometry. Bull. Soc. Math. Belg. Sér. A, 42 (1990), 607-642.

[27] D. Yau. Gerstenhaber structure and Deligne's conjecture for Loday algebras. J. Pure Appl. Algebra, 209 (2007), 739-752.

[28] D. Yau. (Co)homology of triassociative algebras. Int. J. Math. Math. Sci., 2006 (2006), Article ID 69248, 21 pages.

Received March 08, 2008,

Revised May 02, 2008 